\title{
Epigenetic Inactivation of Acetyl-Co A acetyltransferase 1 promotes the proliferation and metastasis of nasopharyngeal carcinoma via decreasing ketogenesis
}

Yunliang Lu

Guangxi Medical University

Xiaohui Zhou

Guangxi Medical University

Weilin Zhao

Guangxi Medical University

Zhipeng Liao

Guangxi Medical University

Bo Li

Guangxi Medical University

Yanping Yang

Guangxi Medical University

Xuemin Zhong

Guangxi Medical University

Yingxi Mo

Guangxi Medical University

Ping Li

Guangxi Medical University

Guangwu Huang

Guangxi Medical University

Xue Xiao

Guangxi Medical University

Zhe Zhang

Guangxi Medical University

Xiaoying Zhou ( $\nabla$ zhouxiaoying1982@outlook.com )

Guangxi Medical University

Research 
Keywords: nasopharyngeal carcinoma, ACAT1, metastasis, EMT, ketone body metabolism

Posted Date: September 8th, 2020

DOI: https://doi.org/10.21203/rs.3.rs-69360/v1

License: (c) (i) This work is licensed under a Creative Commons Attribution 4.0 International License. Read Full License 


\section{Abstract \\ Background}

Acy1 Coenzyme A Acyltransferases 1 (ACAT1) is a key enzyme in the metabolism of ketone bodies, but its expression and biological function in the pathogenesis of NPC remains underexplored.

\section{Methods}

The mRNA and protein expression levels of ACAT1 in NPC and normal control tissues were analyzed by qPCR and immunohistochemistry staining, respectively. GEO database was applied for meta-analysis of ACAT1 mRNA expression and DNA promoter methylation. The role of ACAT1 in NPC proliferation was examined by CCK8 and colony formation assays in vitro and tumorigenicity in vivo. The wound healing and transwell assays were used for analyzing the migratory and invasive ability. cDNA microarray analysis was performed to identify the genes involved in epithelial-mesenchymal transition and dysregulated by ACAT1. These changes were further confirmed by western blot.

\section{Results}

We found that ACAT1 is inactivated in NPC cell lines and primary tissues. DNA microarray data showed higher methylation in the $\mathrm{CpG}$ island region of ACAT1 in NPC than normal tissues. The demethylating reagent 5-aza-dC significantly restored the transcription of ACAT1 in NPC cell lines, suggesting that ACAT1 was inactivated by DNA promoter hypermethylation. Ectopic overexpression of ACAT1 remarkably suppressed the proliferation and colony formation of NPC cells in vitro. As well, the tumorigenesis of NPC cells overexpressing ACAT1 was decreased in vivo. In addition, the migratory and invasive capacities of NPC cells was inhibited by ACAT1 overexpression. Importantly, the higher level of ACAT1 was accompanied by an increased expression of $\mathrm{CDH} 1, \mathrm{EPCAM}$, and a decreased expression of vimentin and SPARC. This strongly indicates that ACAT1 is able to affect the epithelial-mesenchymal transition in NPC, thereby controlling cellular motility. In addition, we found that ACAT1 expression increases the intracellular level of $\beta-\mathrm{HB}$. Moreover, exogenous $\beta$-HB remarkably inhibits the growth of NPC cells in a dose-dependent manner.

\section{Conclusions}

We have discovered that the ketone body metabolism enzyme ACAT1 is epigenetically downregulated in NPC and acts as a potential tumor suppressor in NPC. Our findings highlight the possibility of using the modulation of ketone body metabolism as effective adjuvant therapy for NPC.

\section{Background}


Aberrant energy metabolism was defined as one of the hallmarks of cancer (1). The metabolic reprogramming in cancer includes the generation of additional energy, providing more substrates for biosynthesis, and rebalancing cellular redox status (2), thus facilitating the adaption of tumor cells to the tumor microenvironment and generating extra energy to sustain their malignant growth (3-6).

Nasopharyngeal carcinoma (NPC) derives from the tissue of the nasopharynx. It is the most common one of the head and neck cancer prevalent in southern China and Southeast Asia (7). Synergistic effects of genetic susceptibility, Epstein-Barr virus (EBV) infection, and environmental carcinogens are considered to be the main etiologic factors of NPC (8). More than $75 \%$ of NPC patients were at an advanced stage when diagnosed in the clinic (9). The intensity-modulated radiotherapy (IMRT) has improved the disease control of NPC, but its effect on local recurrence and distant metastasis is limited (10). Therefore, further insights into the carcinogenesis of NPC are necessary to develop new therapeutic approaches.

In the previous study, we found that lipid droplets (LDs) were accumulated in NPC, and the amount of intracellular LDs is associated with the growth of NPC (11), indicating the existence of aberrant lipid metabolism. As raw material for lipid synthesis, Acetyl-CoA is produced during ketone body metabolism, which is a central node in physiological homeostasis. Recently, we reported that a ketone body biosynthesis enzyme, 3-hydroxybutyrate dehydrogenase type $2(\mathrm{BDH} 2)$, was significantly inactivated in NPC (12). In addition, an essential rate-limiting enzyme in ketogenesis, hydroxymethylglutaryl-CoA lyase (HMGCL), was found to be downregulated in NPC. Overexpression of HMGCL restores the production of $\beta$-hydroxybutyrate $(\beta-\mathrm{HB})$, the main component of ketone bodies, thereby impeding NPC cell proliferation and metastasis (13). Our data highlights a remarkably reprogrammed ketone body metabolism in NPC, which warrants further investigation. The enzymes responsible for this might be considered as novel candidate targets for NPC therapy.

Acetyl-CoA acetyltransferase 1 (ACAT1) is located mitochondria and catalyzes the reversible formation of acetoacetyl-CoA from two molecules of acetyl-CoA, as the first step of ketogenesis. To date, most studies have focused on mutations of ACAT1 leading to $\beta$-ketothiolase deficiency (14). Publications about the dysregulation of ACAT1 and its role in tumor pathogenesis are rare. The expression of ACAT1 was decreased in clear cell renal cell carcinoma (ccRCC) (15). Restoring its expression significantly reduces the malignant behavior of ccRCC cells in vitro(16), suggesting that ACAT1 might be a tumor suppressor. On the contrary, as an effective diagnostic and prognostic biomarker, ACAT1 was elevated in aggressive prostate cancer $(17,18)$. However, the role of ACAT1 in NPC remains unclear.

In this study, we investigated the expression of ACAT1 in NPC, bring to light the role of ACAT1 in the biological behavior of NPC and the underlying molecular mechanisms relating to ketone body metabolism.

\section{Methods}

\section{Cell lines and tissue samples}


NPC cell lines (C666-1, HONE1, CNE1, HK1, 5-8F, 6-10B, and TW03) were maintained in DMEM medium (Invitrogen, USA) containing 10\% fetal bovine serum (Invitrogen, USA), $100 \mathrm{U} / \mathrm{mL}$ penicillin, and 100 $\mu \mathrm{g} / \mathrm{mL}$ streptomycin in a humidified incubator with $5 \% \mathrm{CO}_{2}$ at $37^{\circ} \mathrm{C}$.

In all, primary NPC tumor tissues were obtained from 42 diagnosed and untreated cases with informed consent from the donors in the Department of Otolaryngology-Head and Neck Surgery, First Affiliated Hospital of Guangxi Medical University (Nanning, China). The diagnoses were established by experienced pathologists according to the World Health Organization (WHO) classification. We also included 36 normal nasopharyngeal epithelial tissues as control.

\section{Antibodies, Plasmids, and Transfection}

The information of antibodies was as followed: ACAT1 (1:1000, HPA004428, Sigma, USA), $\beta$-catenin (1:1000, sc-376841, Santa Cruz, USA) and E-cadherin (1:1000, \#3195P), Vimentin (1:1000, \#5741P) and GAPDH (1:10000 \#5174P) were purchased from Cell Signaling Technology.

The demethylating reagent 5-aza-dC was purchased from Sigma (\#A3656, USA). Full-length cDNA from the open reading frame of ACAT1 (Origene, USA) was subcloned into the pCMV6-Entry vector (Origene, USA). NPC cells were cultured in 6-well dishes to 70-90\% confluence then transfected with $2 \mu \mathrm{g}$ pCMV6Entry or ACAT1 plasmid using an X-treme GENE HP DNA Transfection Reagent (Roche, Germany) for 48 hrs.

\section{Real-time RT-PCR}

Briefly, first-strand complementary DNA was synthesized using a First-Stand Reverse Transcription System (Transgene, Beijing, China). Real-time RT-PCR was carried out using SYBR Green PCR master mix in Step One Plus System (Applied Biosystems, USA). The primer sequences and cycling conditions for all experiments were as follows, ACAT1-F 5'-GGCTGGTGCAGGAAATAAGA-3', ACAT1-R 5' GGAATCCCTGCCTTTTCAAT-3'; GAPDH-F 5'-GCTCAGACACCATG-GGGAAG-3', GAPDH-R 5'TGTAGTTGAGGTCAATGAAGGGG-3'. Empty vector-transfected cells were used as control. The relative gene expression was calculated using the comparative threshold cycle $\left(2^{-\triangle \triangle C T}\right)$ equation. All the experiments were performed in triplicate.

\section{Immunohistochemical staining}

After deparaffinization and rehydration, the antigen was retrieved in $5 \%$ urea buffer by microwave heating for $5 \mathrm{~min}$, and then incubated in $3 \% \mathrm{H}_{2} \mathrm{O}_{2}$ for 30 min to block endogenous peroxidase activity. Tissue sections were incubated with the first antibody overnight at $4{ }^{\circ} \mathrm{C}$ and followed by a secondary antibody for $1 \mathrm{~h}$ at room temperature. Subsequently, 3,3'-diaminobenzidine (DAB) reagent (ZLI-9018, ZSGB-BIO, Beijing) was used for peroxidase reaction and hematoxylin was used for counterstaining. Images were acquired under a microscope (Olympus C-5050, Japan).

\section{Western blot}


Total protein was isolated using RIPA buffer (BeyotimeBiotechnology, China) containing a protease inhibitor cocktail (Fabio Science, China). Equal amounts of protein $(50 \mu \mathrm{g})$ were separated by electrophoresis on a 4-12\% SDS-PAGE gel and transferred to Nitrocellulose Membrane (Thermo Fisher Scientific, USA), which were blocked in $5 \%$ bovine serum albumin (BSA) in $1 \times$ Tris-buffered saline with $0.1 \%$ Tween-20. The membranes were subsequently blocked in $5 \%$ BSA and incubated with primary antibodies overnight at $4^{\circ} \mathrm{C}$. Subsequently, secondary antibodies (anti-rabbit/mouse, 1:10000, 92632211/926-68070, Licor, USA) were used at room temperature for $2 \mathrm{hrs.} \mathrm{Fluorescent} \mathrm{signals} \mathrm{were}$ captured by LI-COR Odyssey (Li-Cor, USA).

\section{Cell proliferation assay}

$1 \times 10^{3}$ cells were seeded in each well of 96-well plates. $10 \mu$ l CCK-8 (Dojindo, Japan) were added to each well following by incubation for the indicated time periods (1, 2, 3, 4, and 5 days). The absorbance $(\mathrm{OD} 450 \mathrm{~nm})$ was measured in a microplate reader (BioTek, USA).

\section{In vivo tumorigenesis}

The animal study was approved by the Animal Ethical Committee of First Affiliated Hospital of Guangxi Medical University. All the methods were carried out in accordance with the approved guidelines. Five 5week-old male BALB/c-nu nude mice (Vital River Laboratory Animal Technology, China) were injected with $1 \times 10^{6}$ pCMV6-Entry-5-8F/HONE1 cells in the right flank and an equal amount of ACAT1-5-8F/HONE1 cells was injected into the left flank. The tumor volume was assessed by 2D measurements at $3,6,9,12,15$, 18 days. Tumor volume was calculated as volume $\left(\mathrm{mm}^{3}\right)=$ length $\times$ width $\times 0.5$. Eighteen days for $5-8 \mathrm{~F}$ and fifteen days for HONE1 after inoculation, all mice were killed and tumors were removed.

\section{cDNA microarray analysis}

Affymetrix GeneChip Genome U133 Plus 2.0 expression array and Gene-Cloud of Biotechnology Information (GCBI) platform (www.gcbi.com.cn) were used to analyze genes with differential expression between ACAT1-5-8F and pCMV6-Entry-5-8F cells. The microarray data is available via the following accession identifier on the NCBI-GEO database: GSE155206.

\section{Wound healing}

Cells $\left(8 \times 10^{5}\right.$ per well) were seeded into 12 -well plates in DMEM medium without FBS overnight. Monolayer cells were scratched by an ibidi Culture-Insert (No. 80209, ibidi, Germany). Images were acquired under an inverted phase microscope (TS100, Nikon, Japan) at $0 \mathrm{~h}$ and $24 \mathrm{hrs}$. The migratory distance was analyzed by Image J ver.1.51k (NIH, USA) software.

\section{Transwell}

Cells $\left(7 \times 10^{4}\right.$ per well) suspended in $200 \mu$ of serum-free medium were seeded in the upper transwell chamber (8 mm pores, Corning, USA), pre-coated with matrigel (BD Biosciences). The lower chamber was 
filled with DMEM medium with $10 \%$ FBS. Non-invading cells were removed by using a cotton-tipped swab after $36 \mathrm{hrs}$. Invasive cells on the lower membrane surface were fixed with 1\% paraformaldehyde, stained with $0.5 \%$ crystal violet, and photographed.

\section{$\beta$-hydroxybutyrate ( $\beta$-HB) detection}

Cells were grown in 60-mm dishes in serum-free DMEM medium for $48 \mathrm{hrs}$ and then lysed with RIPA buffer. $\beta$-HB colorimetric assay kit (\#700190, Cayman Chemical, Ann Arbor, MI, USA) was used for determining the intracellular $\beta$-HB level as previously (19).

\section{Statistical analysis}

All data were analyzed using SPSS 20.0 (SPSS Inc., Chicago, IL, USA). Data are expressed as mean \pm SD and were analyzed by Pearson's chi-square test and Fisher's exact test. Statistical significance was considered at $* p<0.05, * * p<0.01$ and ${ }^{* \star *} p<0.01$.

\section{Results}

\section{The expression of ACAT1 is significantly downregulated in NPC.}

To reveal the alteration of ACAT1 in NPC, we firstly investigated the transcriptional level of ACAT 1 in seven NPC cell lines (HK1, HONE1, CNE1, 5-8F, 6-10B, and C666-1) compared with an immortalized normal nasopharyngeal epithelial cell line (NP69). Figure 1A shows a reduction of ACAT1 in NPC cell lines. We further analyzed both mRNA and protein expression of ACAT1 in NPC tissues and non-cancer control tissues. We found that the transcription of the ACAT1 gene was significantly downregulated in 21 NPC primary tumors, but easily detected in all 23 NNE samples (Fig. 1B). In order to evaluate the consistency of abnormal transcription of ACAT1 in NPC, we performed a meta-analysis using 6 sets of microarrays from the GEO database including 143 NPC tissues and 42 normal tissues (Table S1). The results generated by the random-effects model indicated that significant heterogeneity existed among individual datasets $\left(I^{2}=61.2 \%, p=0.025\right)$ and the pooled Standard Mean Difference (SMD) as $-0.98(95 \%$ Cl: $-1.63,-0.33$, Fig. S1A). No significant difference was found in the sensitivity analysis (Fig. S1B). The results of Begg's test showed no significant publication bias ( $p=0.452$, Fig. S1C ).

Besides, we also found that the mRNA level of ACAT1 in head and neck squamous carcinoma (HNSC) was decreased in contrast with normal control tissues based on the TCGA database (Fig. S1), which is in line with our findings in NPC. The lower mRNA expression of ACAT1 was positively correlated with HNSC patients older than 60 years, and higher histologic grade (Table S2).

In addition, we measured the protein levels of ACAT1 in NPC tissue and NNE tissues. ACAT1 protein was localized in the cytoplasm of cells and was highly expressed in the NNE layer $(n=19)$ (Fig. 2), while almost absent in NPC tissue $(n=15)$. This finding further supports that ACAT1 is inactivated in NPC. 
To address the mechanism for the inactivation of ACAT1 in NPC, we firstly analyzed the miRNAs potentially targeting ACAT1, including miR-21, miR-23b, miR-1260, and miR-9, which were screened by the Gene-Cloud of Biotechnology Information platform (Fig. S3A). However, we could not find miRNAs that were significantly overexpressed in primary NPC tissues as compared to normal control samples based on the miRNA microarray database (GSE32960) (Fig. S3B). The expression of miR-21 and miR-1260 is even decreased significantly in NPC samples.

Interestingly, we found that a CpG island with a length of $241 \mathrm{bp}(-295 \mathrm{bp} \rrbracket-55 \mathrm{bp}$ from the transcription starting site) was found in the DNA promoter region of ACAT1. We analyzed the methylation microarray dataset (GSE62336) and found that the methylation modification in the CpG island region of ACAT1 is remarkably heavier in NPC $(n=25)$ in contrast to NNE $(n=25)$ tissues (Fig. 3A). In addition, the mRNA level of ACAT1 was restored significantly upon treatment with the demethylation reagent 5-aza-dC in the NPC cell lines 5-8F, HONE1, and TW03. The mRNA level of ACAT1 was elevated in HK1 by 5-aza-dC treatment as well, but no significant difference was observed (Fig. 3B). These data suggest that ACAT1 might be inactivated in NPC by DNA promoter hypermethylation.

Furthermore, we found that the level of methylation in the promoter region of the ACAT1 gene is higher also in HNSC, based on the TCGA database (Fig. S4A). Importantly, the transcriptional level of ACAT1 is inversely correlated with the methylation level in its promoter, which strongly supports hypermethylation as the reason for ACAT1 inactivation.

Overexpression of ACAT1 suppresses cell proliferation and colony formation of NPC cell lines in vitro.

To investigate the biological function of ACAT1 in NPC, we established 5-8F and HONE1 cell lines stably overexpressing of ACAT1. Western blot results confirmed that ACAT1 was successfully expressed in both cell lines (Fig. 4A). The proliferative rate of ACAT1-5-8F/HONE1 cells was remarkably slower than the control cells (Fig. 4B). Also, the capacity of colony formation of both NPC cell lines was reduced significantly by ACAT1 overexpression(Fig. 4C). Therefore, our finding indicates that overexpression of ACAT1 suppresses the growth of NPC cells in vitro.

\section{ACAT1 suppresses NPC tumor formation in vivo.}

To confirm the suppressive effect of ACAT1 expression on NPC tumor cell growth, we examined the tumorigenesis of ACAT1-5-8F/HONE1 and pCMV6-Entry-5-8F/HONE1 in vivo. We observed that the average volume of tumor-derived from ACAT1-5-8F/HONE1 is lower than that from pCMV6-Entry-58F/HONE1 (Fig. 5). However, a statistical significance was observed only in the 5-8F cell line.

\section{ACAT1 suppresses NPC cell migration and invasion via epithelial-mesenchymal transition.}

We further addressed the effect of ACAT1 on migratory and invasive capacities in NPC cells. The wound healing assays revealed a slower gap closure in ACAT1-5-8F and ACAT1-HONE1 cells in contrast to control cell lines (Fig. 6A). In addition, the number of invading ACAT1-5-8F and ACAT1-HONE1 cells was 
lower than for the control cell lines (Fig. 6B). Our finding suggests that overexpression of ACAT1 attenuates the migration and invasion of NPC cells.

To investigate the potential molecular mechanism by which ACAT1 affects migratory and invasive capacity, we performed cDNA microarray analysis in ACAT1-5-8F and PCMV6-Entry-5-8F cells. We found that genes involved in epithelial-mesenchymal transition (EMT) were significantly altered by ACAT1 overexpression, including downregulation of SPARC, ZEB2, etc. and upregulation of EPCAM, CDH1, and $\mathrm{CDH} 3$, etc. (Fig. $6 \mathrm{C}$ ). In addition, we analyzed the expression of E-cadherin and vimentin by western-blot. In comparing with control cell lines, E-cadherin was upregulated in ACAT1-5-8F/HONE1 cells, while vimentin was downregulated (Fig. 6D). Therefore, overexpression of ACAT1 may reverse the EMT process, thereby reducing the metastatic potential of NPC cells.

\section{ACAT1 increases the intracellular level of $\beta$-hydroxybutyrate ( $\beta$-HB) in NPC cells.}

To understand the functional effect of ACAT1 on ketogenesis, we assessed the relative concentration of intracellular $\beta-\mathrm{HB}$, the main component of ketone bodies, in ACAT1-5-8F/HONE1 and Ctrl-5-8F/HONE1 cells. The intracellular $\beta-\mathrm{HB}$ level was significantly higher in ACAT1-5-8F/HONE1 than in Ctrl-5-8F/HONE1 (Fig. 7A). As we previously found(13), a higher concentration of intracellular $\beta$-HB suppresses the proliferation of HONE1 and 5-8F cells in a dose-dependent manner (Fig. 7B). Thus, overexpression of ACAT1 might reverse the malignant phenotype of NPC cells by elevating $\beta-\mathrm{HB}$.

\section{Discussion}

Functioning as a kind of high-energy mitochondrial fuels, ketone bodies are normally generated in hepatocytes and used during starvation (20). Recent studies provide evidence that ketone bodies can be produced in cancer cells as well as in tumor stroma $(19,21)$. In this study, we show that the enzyme ACAT1 catalyzing the first step of ketogenesis is inactivated in NPC cell lines and primary tumor tissues. This further supports our previous finding that the production of ketones in NPC is inhibited. On the other hand, ACAT 1 is also required for the utilization of ketone bodies. This suggests that NPC cells tend to provide and utilize the energy for promoting growth in other sources than ketones. Thus, a significant ketone body metabolism-inactivation pattern is one of the hallmarks of NPC. Besides ACAT1, the other two key enzymes BDH1 and OXT1 were all downregulated in Glioblastoma (22). The expression of ACAT1 is regulated by the well-studied onco-microRNA miR-21, which is elevated in various tumors (23). We found a $\mathrm{CpG}$ island in the promoter region of ACAT1. Based on the methylation microarray data from the GEO database, the methylation level of this $\mathrm{CpG}$ island region is significantly higher in NPC tissues in contrast to normal control tissues. In addition, 5-aza-dC treatment upregulated the mRNA level of ACAT1, further indicating that ACAT1 is inactivated by DNA hypermethylation in NPC. To our knowledge, this is a novel mechanism for modulating ACAT1 expression in tumors. The upregulation of ACAT1 in breast cancer was shown to induce apoptosis(23). We found that restoring the expression of ACAT1 leads to increased intracellular $\beta$-hydroxybutyrate $(\beta-\mathrm{HB})$ while the proliferation, colony formation, and in vivo tumorigenesis of NPC cells was decreased remarkably. In addition, extracellular $\beta$-HB impedes the growth 
of NPC cells as well. This directly supports the opinion that ketones interfere with NPC cell survival. Previously it was shown that both intracellular and extracellular $\beta$-HB induce significant generation of ROS, thereby suppressing the viability of NPC (19). As an important factor for tumorigenesis, chronic inflammation promotes tumor development, progression, and metastatic dissemination, as well as treatment resistance. $\beta$-HB was identified as the factor exerting an anti-inflammatory effect by inhibiting the inflammasome NOD-like receptor pyrin domain-containing 3 (NLRP3) $(24,25)$. In glioma, inhibiting the inflammatory microenvironment suppresses tumor cell migration upon treatment with $\beta$-HB, implying that $\beta-H B$ could be of benefit for glioma therapy (26). In accordance with our study, increasing the $\beta-H B$ level by overexpressing ACAT1 substantially reduces the motility of NPC cells. 3-HydroxymethylglutarylCoA synthase 2 (HMGCS2) is the enzyme directly downstream of ACAT1 in the ketogenesis pathway (27). Its inactivation in hepatocellular carcinoma has been verified and was positively associated with ketone body production. Importantly, the ketone bodies inhibit tumor cell migration by reversing epithelialmesenchymal transition (EMT) signaling (28). Similarly, we showed that the epithelial markers (CDH1 and EPCAM) were increased in NPC cells after overexpressing ACAT1, while mesenchymal markers (vimentin and SPARC) were decreased. Our data indicate that ACAT1 represses the movement of NPC cells by regulating the expression of EMT-related markers.

In order to further uncover the mechanisms underlying ACAT1-mediated control of

The EMT signaling, we focused on the expression of $\mathrm{CDH} 1$, which is upregulated by overexpressing ACAT1 or by $\beta$-HB treatment alone (19). As an endogenous histone deacetylase inhibitor (HDACi), $\beta-\mathrm{HB}$ is able to regulate gene expression affecting several cellular processes(29). We observed increased acetylation of lysine 9 and lysine 14 of histone 3 in the promoter region of $\mathrm{CDH} 1$ after the treatment with $\beta-\mathrm{HB}$, indicating transcriptional stimulation of $\mathrm{CDH} 1$ (data not shown). HDACi induced p53-dependent apoptosis in NPC cells (30) and retarded the growth of carcinomas of the cervix, colon, and rectum in vitro (31). Surprisingly, a recent study reported that NPC cells were turned into a mesenchymal cell phenotype after short-term stimulation with the HDACi trichostatin A. However, an invasive phenotype was not induced (32). Additionally, HDACs contribute to maintaining EBV latent infection (33). HDACs, such as TSA, SAHA, and butyric acids, act as potent inducers of EBV reactivation, as well as mediating apoptosis of NPC cells, Therefore, reactivating EBV by HDACi is a potential approach to treat NPC. So far, it remains unknown if EBV reactivation in NPC cell lines can be induced by $\beta$-HB. Further verification is necessary.

In fact, as non-toxic adjuvant therapy, ketogenic diets or ketone supplementation have shown a positive therapeutic advantage in malignancies. Notably, the preclinical studies demonstrate that a ketogenic diet increases the radiation sensitivity in xenograft models of pancreatic cancer (34). Our previous and present findings show that overexpressing the ketogenesis genes HMGCL and ACAT1, resulted in increased intracellular $\beta$-HB in both renal cell carcinoma and NPC $(16,19)$, thereby inhibiting the growth capacity of tumor cells. As well, exogenous $\beta-H B$ remarkably suppresses the proliferation and metastasis of NPC cells. Thus, elevating the level of $\beta-\mathrm{HB}$ by targeting ketogenic genes, ketogenic diets or ketone 
supplementation might be a promising therapeutic approach for NPC patients. This is worthy of further confirmation in animal models.

\section{Conclusions}

In summary, we discovered that the expression of ACAT1 was inactivated in NPC due to promoter hypermethylation. Overexpression of ACAT1 elevates the intracellular $\beta$-HB in NPC cells, inhibits the proliferation, migration, and invasive growth of NPC cells. Our data reveal that epigenetic modification affects the ketone body metabolism which might contribute to pathogenesis of NPC. In addition, our findings support the manipulation of ketone body metabolism, for example, in a form of a drug or by dietary intervention is a potentially valuable approach for the prevention and treatment of NPC.

\section{Abbreviations}

5-aza-dC: 5-aza-2'-deoxycytidine; ACAT1:Acetyl-Co A acetyltransferase 1; BDH2:3-hydroxybutyrate dehydrogenase type 2; $\beta$-HB: $\beta$-hydroxybutyrate; EBV:Epstein-barr virus; EMT:epithelial-mesenchymal transition; HDACi:histone deacetylases inhibitor; HMGCL:hydroxymethylglutaryl-CoA lyase; NPC:nasopharyngeal carcinoma.

\section{Declarations}

\section{Availability of data and materials}

All data generated or analyzed during this study are included in this published article and its supplementary information files. The microarray data is available via the following accession identifier on the NCBI-GEO database: GSE155206.

\section{Ethics approval and consent to participate}

This study was granted by the Research Ethics Committee of the First Affiliated Hospital of Guangxi Medical University, China (documents no.2016-KY-050).

\section{Consent for publication}

All authors have agreed to the publication of the article.

\section{Competing interests}

The authors declare no competing or financial interests.

\section{Funding}


This work was supported by grants from the National Natural Science Foundation of China ( 81460412 , 81772882, 81660458, 81660445).

\section{Authors' contributions}

$X Y Z$ and $Z Z$ designed the experiments. BL and ZPL analyzed the data. $L Y L, X H Z$ and WLZ wrote the manuscript. GWH directed the research. YPY, $X M Z$, and $L Y L$ performed the experiments. $X X, Y X M$ and $P L$ provided research materials and methods. All authors read and approved the final manuscript.

\section{Acknowledgments}

We appreciate Dr. Liudmila Matskova and Dr. Gösta Winberg from Karolinska Institutetgive, Sweden for useful comments.

\section{References}

1. Hanahan D, Weinberg RA. Hallmarks of cancer: the next generation. Cell. 2011;144(5):646-74.

2. Zhou W, Mukherjee P, Kiebish MA, Markis WT, Mantis JG, Seyfried TN. The calorically restricted ketogenic diet, an effective alternative therapy for malignant brain cancer. Nutr Metab (Lond). 2007;4:5.

3. Zhang ZP, Tang HL, Wang ZY, Zhang BX, Liu W, Lu HM, et al. MiR-185 Targets the DNA Methyltransferases 1 and Regulates Global DNA Methylation in human glioma. Molecular Cancer. 2011;10.

4. Chang CH, Qiu J, O'Sullivan D, Buck MD, Noguchi T, Curtis JD, et al. Metabolic Competition in the Tumor Microenvironment Is a Driver of Cancer Progression. Cell. 2015;162(6):1229-41.

5. Lee N, Kim D. Cancer Metabolism: Fueling More than Just Growth. Mol Cells. 2016;39(12):847-54.

6. Tarrado-Castellarnau M, de Atauri $\mathrm{P}$, Cascante $\mathrm{M}$. Oncogenic regulation of tumor metabolic reprogramming. Oncotarget. 2016;7(38):62726-53.

7. Chang ET, Adami HO. The enigmatic epidemiology of nasopharyngeal carcinoma. Cancer Epidemiol Biomarkers Prev. 2006;15(10):1765-77.

8. Tsao SW, Yip YL, Tsang CM, Pang PS, Lau VM, Zhang G, et al. Etiological factors of nasopharyngeal carcinoma. Oral Oncol. 2014;50(5):330-8.

9. Chua MLK, Wee JTS, Hui EP, Chan ATC. Nasopharyngeal carcinoma. Lancet. 2016;387(10022):1012-24.

10. Kawashima M, Ariji T, Kameoka S, Ueda T, Kohno R, Nishio T, et al. Locoregional control after intensity-modulated radiotherapy for nasopharyngeal carcinoma with an anatomy-based target definition. Jpn J Clin Oncol. 2013;43(12):1218-25. 
11. Zhou XY, Wei JZ, Chen F, Xiao X, Huang TT, He Q, et al. Epigenetic downregulation of the ISG15conjugating enzyme $\mathrm{UbcH} 8$ impairs lipolysis and correlates with poor prognosis in nasopharyngeal carcinoma. Oncotarget. 2015;6(38):41077-91.

12. Li B, Liao Z, Mo Y, Zhao W, Zhou X, Xiao X, et al. Inactivation of 3-hydroxybutyrate dehydrogenase type 2 promotes proliferation and metastasis of nasopharyngeal carcinoma by iron retention. British journal of cancer. 2020;122(1):102-10.

13. Luo WQ, Qin LT, Li B, Liao ZP, Liang JZ, Xiao XL, et al. Inactivation of HMGCL promotes proliferation and metastasis of nasopharyngeal carcinoma by suppressing oxidative stress. Sci Rep-Uk. 2017;7.

14. Abdelkreem E, Harijan RK, Yamaguchi S, Wierenga RK, Fukao T. Mutation update on ACAT1 variants associated with mitochondrial acetoacetyl-CoA thiolase (T2) deficiency. Hum Mutat. 2019;40(10):1641-63.

15. Zhao ZH, Wu F, Ding ST, Sun L, Liu Z, Ding KJ, et al. Label-free quantitative proteomic analysis reveals potential biomarkers and pathways in renal cell carcinoma. Tumor Biol. 2015;36(2):939-51.

16. Cui W, Luo W, Zhou X, Lu Y, Xu W, Zhong S, et al. Dysregulation of Ketone Body Metabolism Is Associated With Poor Prognosis for Clear Cell Renal Cell Carcinoma Patients. Front Oncol. 2019;9:1422.

17. Saraon P, Trudel D, Kron K, Dmitromanolakis A, Trachtenberg J, Bapat B, et al. Evaluation and prognostic significance of ACAT1 as a marker of prostate cancer progression. Prostate. 2014;74(4):372-80.

18. Saraon P, Cretu D, Musrap N, Karagiannis GS, Batruch I, Drabovich AP, et al. Quantitative proteomics reveals that enzymes of the ketogenic pathway are associated with prostate cancer progression. Mol Cell Proteomics. 2013;12(6):1589-601.

19. Luo W, Qin L, Li B, Liao Z, Liang J, Xiao X, et al. Inactivation of HMGCL promotes proliferation and metastasis of nasopharyngeal carcinoma by suppressing oxidative stress. Sci Rep. 2017;7(1):11954.

20. Yang H, Shan W, Zhu F, Wu J, Wang Q. Ketone Bodies in Neurological Diseases: Focus on Neuroprotection and Underlying Mechanisms. Front Neurol. 2019;10:585.

21. Martinez-Outschoorn UE, Lin Z, Whitaker-Menezes D, Howell A, Lisanti MP, Sotgia F. Ketone bodies and two-compartment tumor metabolism: stromal ketone production fuels mitochondrial biogenesis in epithelial cancer cells. Cell Cycle. 2012;11(21):3956-63.

22. Vallejo FA, Shah SS, de Cordoba N, Walters WM, Prince J, Khatib Z, et al. The contribution of ketone bodies to glycolytic inhibition for the treatment of adult and pediatric glioblastoma. J Neurooncol. 2020;147(2):317-26.

23. Chanyshev MD, Razumova YV, Ovchinnikov VY, Gulyaeva LF. MiR-21 regulates the ACAT1 gene in MCF-7 cells. Life Sci. 2018;209:173-8.

24. Yamanashi T, Iwata M, Kamiya N, Tsunetomi K, Kajitani N, Wada N, et al. Beta-hydroxybutyrate, an endogenic NLRP3 inflammasome inhibitor, attenuates stress-induced behavioral and inflammatory responses. Sci Rep. 2017;7(1):7677. 
25. Youm YH, Nguyen KY, Grant RW, Goldberg EL, Bodogai M, Kim D, et al. The ketone metabolite betahydroxybutyrate blocks NLRP3 inflammasome-mediated inflammatory disease. Nat Med. 2015;21(3):263-9.

26. Shang S, Wang L, Zhang Y, Lu H, Lu X. The Beta-Hydroxybutyrate Suppresses the Migration of Glioma Cells by Inhibition of NLRP3 Inflammasome. Cell Mol Neurobiol. 2018;38(8):1479-89.

27. Shafqat N, Turnbull A, Zschocke J, Oppermann U, Yue WW. Crystal structures of human HMG-CoA synthase isoforms provide insights into inherited ketogenesis disorders and inhibitor design. $\mathrm{J} \mathrm{Mol}$ Biol. 2010;398(4):497-506.

28. Wang YH, Liu CL, Chiu WC, Twu YC, Liao YJ. HMGCS2 Mediates Ketone Production and Regulates the Proliferation and Metastasis of Hepatocellular Carcinoma. Cancers (Basel). 2019;11(12).

29. Qiu X, Rong X, Yang J, Lu Y. Evaluation of the antioxidant effects of different histone deacetylase inhibitors (HDACis) on human lens epithelial cells (HLECs) after UVB exposure. BMC Ophthalmol. 2019;19(1):42.

30. Yan M, Qian YM, Yue CF, Wang ZF, Wang BC, Zhang W, et al. Inhibition of histone deacetylases induces formation of multipolar spindles and subsequent p53-dependent apoptosis in nasopharyngeal carcinoma cells. Oncotarget. 2016;7(28):44171-84.

31. Anantharaju PG, Reddy DB, Padukudru MA, Chitturi CMK, Vimalambike MG, Madhunapantula SV. Induction of colon and cervical cancer cell death by cinnamic acid derivatives is mediated through the inhibition of Histone Deacetylases (HDAC). Plos One. 2017;12(11).

32. Shen Z, Liao X, Shao Z, Feng M, Yuan J, Wang S, et al. Short-term stimulation with histone deacetylase inhibitor trichostatin a induces epithelial-mesenchymal transition in nasopharyngeal carcinoma cells without increasing cell invasion ability. BMC Cancer. 2019;19(1):262.

33. Herbein G, Wendling D. Histone deacetylases in viral infections. Clin Epigenetics. 2010;1(1-2):13-24.

34. Zahra A, Fath MA, Opat E, Mapuskar KA, Bhatia SK, Ma DC, et al. Consuming a Ketogenic Diet while Receiving Radiation and Chemotherapy for Locally Advanced Lung Cancer and Pancreatic Cancer: The University of lowa Experience of Two Phase 1 Clinical Trials. Radiat Res. 2017;187(6):743-54.

\section{Figures}


A

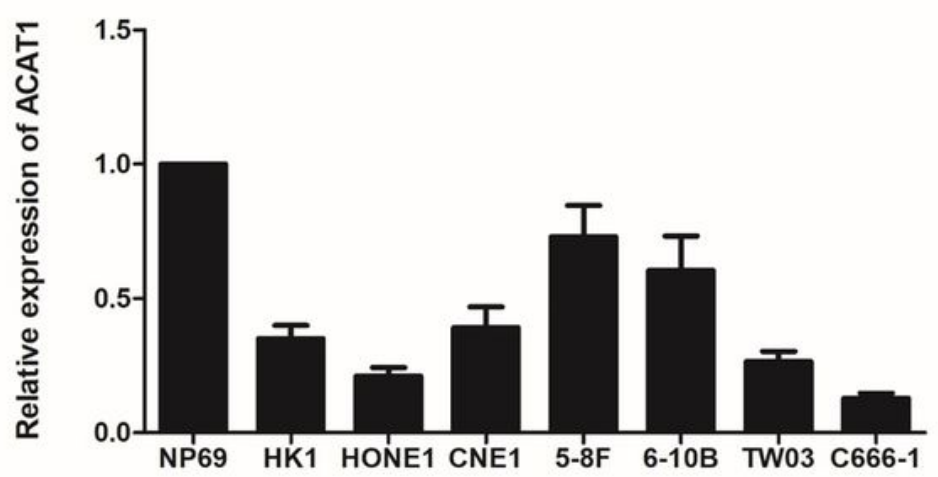

B
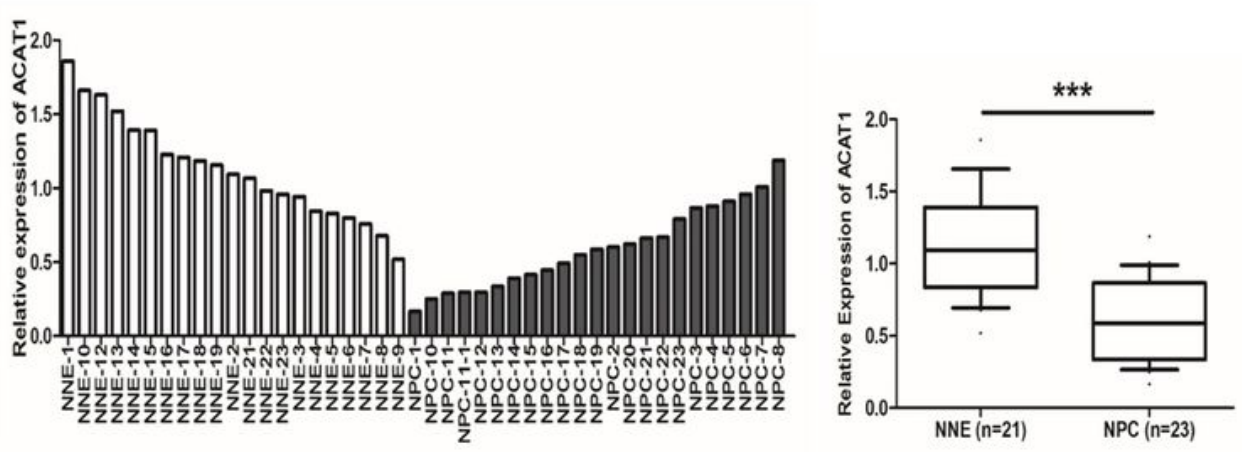

Figure 1

The expression of ACAT1 is downregulated in NPC. (A) ACAT1 mRNA level was determined by real-time RT-PCR in 7 NPC cell lines and a non-cancerous nasopharyngeal epithelial (NNE) cell line NP69. (B) Relative ACAT1 mRNA expression in NPC primary biopsies $(n=23)$ and NNE samples $(n=21)$. The line inside the boxes represents the median value. The box length indicates the interquartile range. $\star \star \star p<$ 0.001 . 

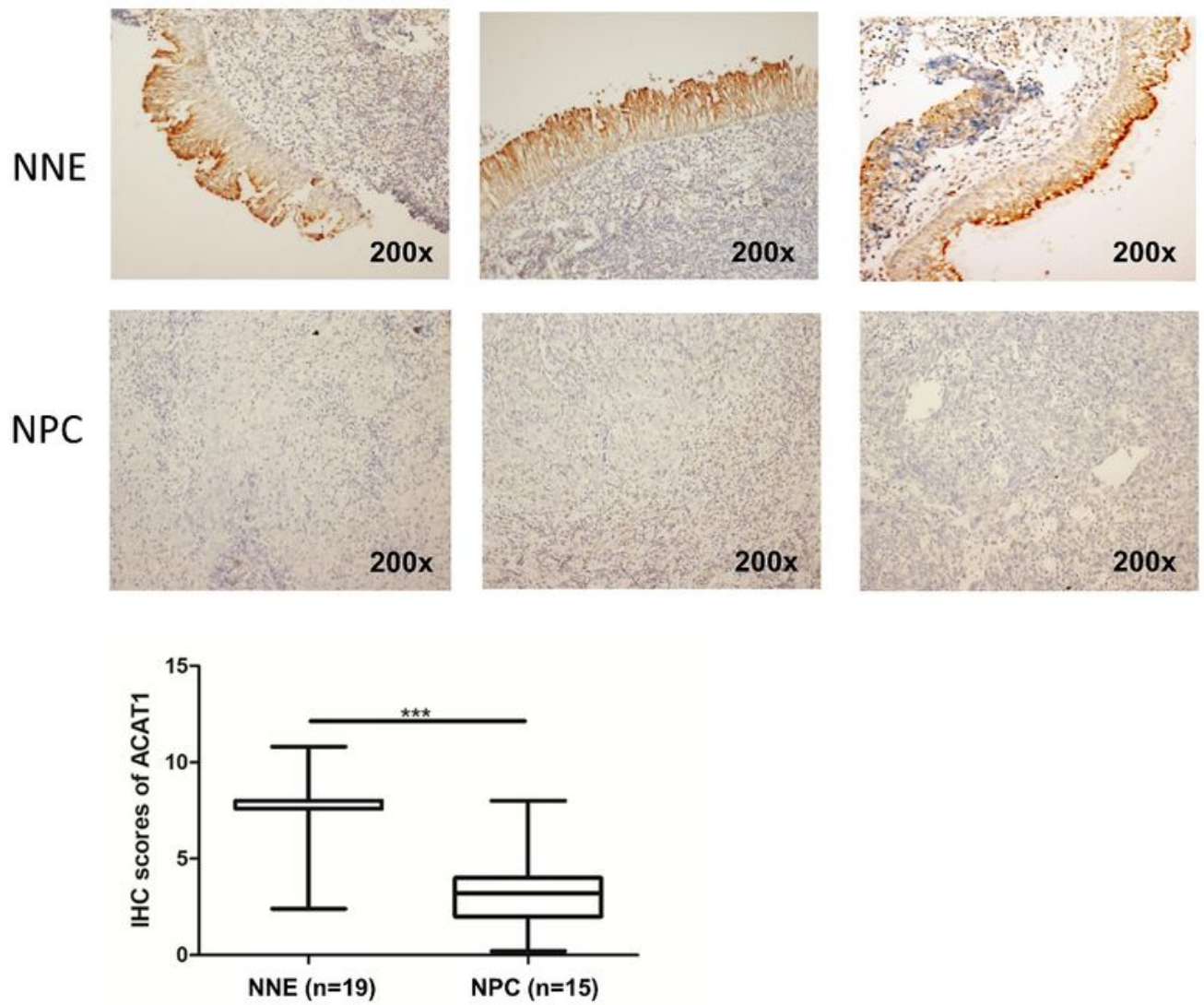

\section{Figure 2}

Immunohistochemical staining of ACAT1 in NNE tissue $(n=19)$ and NPC $(n=15)$. Magnifications $\times 200$. $\star \star \star p p<0.001$. 
A

$P=0.041$

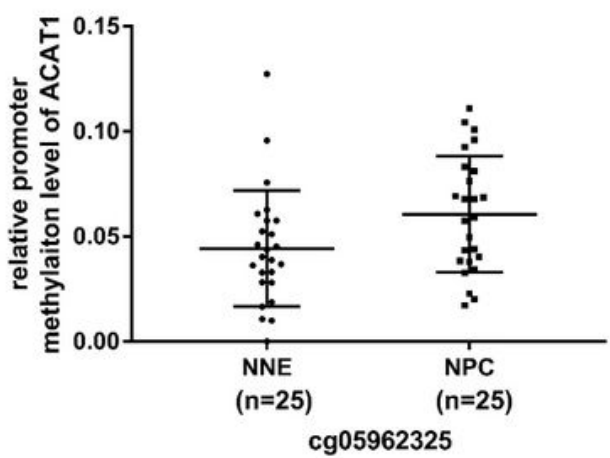

$P=0.015$

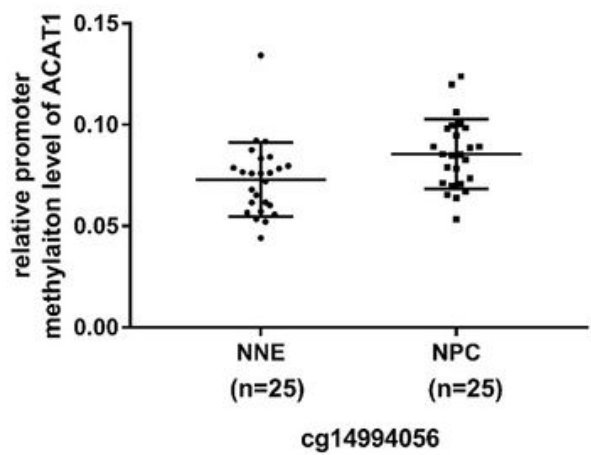

B
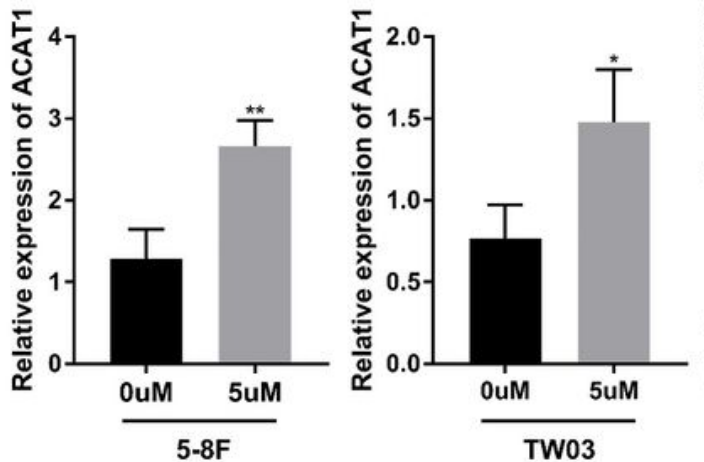

$P=0.005$

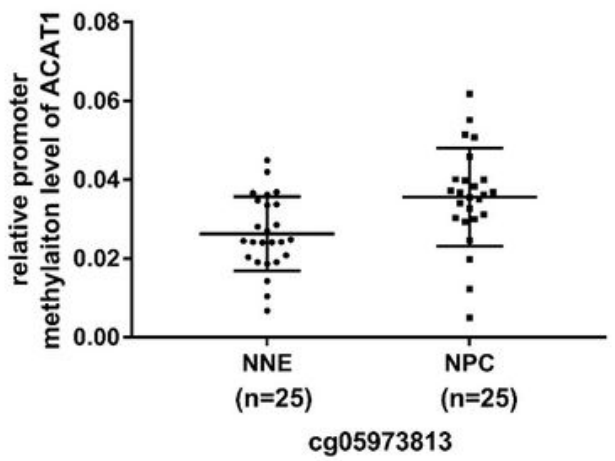

$P=0.006$
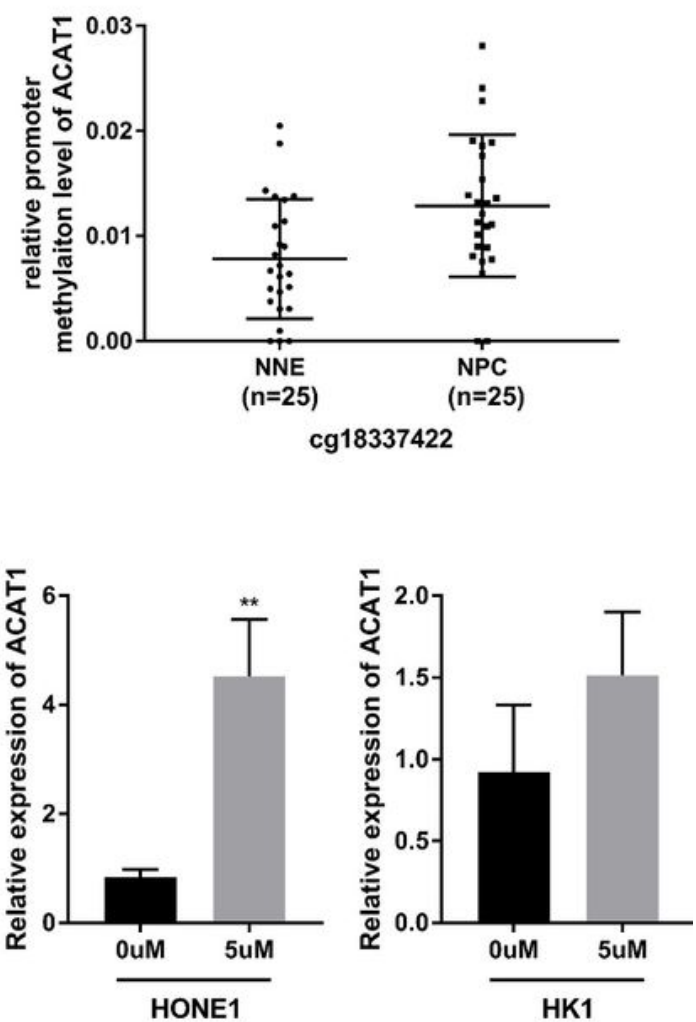

\section{Figure 3}

Hypermethylation of the ACAT1 CpG island in NPC tumor biopsies and normal nasopharyngeal tissue. (A) DNA methylation microarray data (GSE62336) containing 25 cases of NPC primary tissues and 25 cases of normal nasopharyngeal epitheliums was used for analyzing the relative methylation level in the $\mathrm{CpG}$ island of ACAT1 gene. Four probes targeting the CpG island region of ACAT1 were found. The dot plot 
shows the relative methylation level of the ACAT1 gene. (B) Real-time RT-PCR analysis of the mRNA level of ACAT1 in four NPC cell lines with and without treatment with 5-aza-dC, $5 \mu \mathrm{M} / \mathrm{L}$ for 4 days.

A

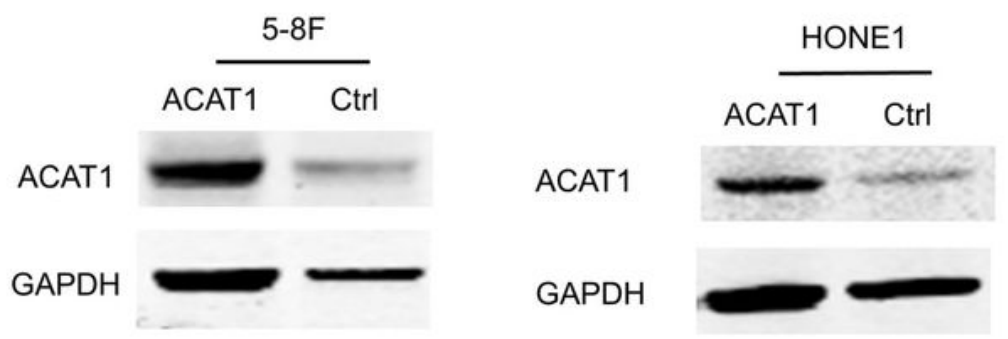

B
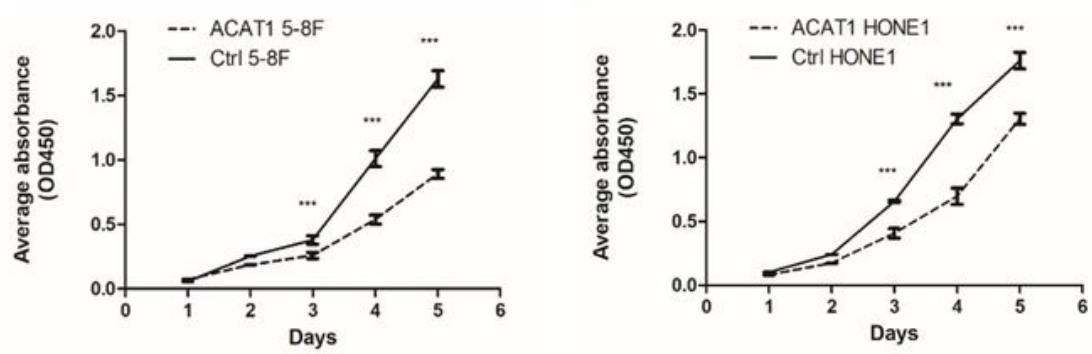

C
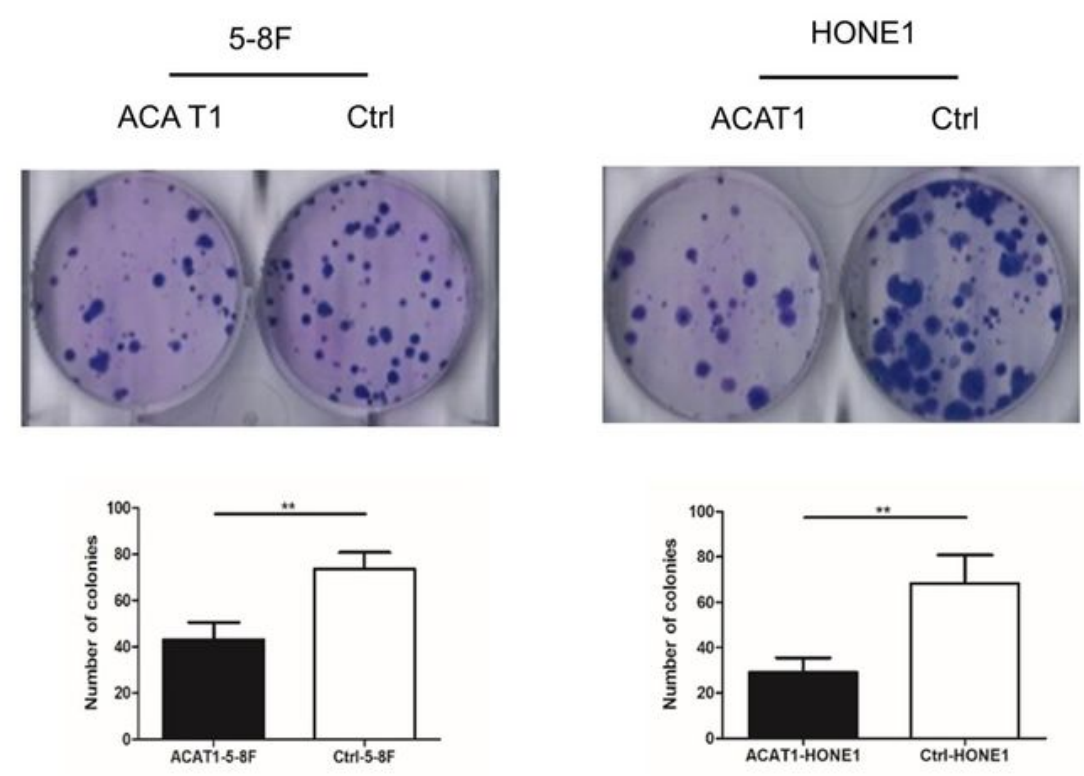

Figure 4

Overexpression of ACAT1 inhibits proliferation. (A) ACAT1 expression in stably transfected $5-8 \mathrm{~F}$ and HONE1 cell lines was confirmed by western blotting. (B) Proliferation of stably ACAT1 transfected 5-8F 
and HONE1 cells determined by CCK-8 assay $(\mathrm{OD}=450 \mathrm{~nm})$. Mean \pm sd.; Student's t-tests; ${ }^{\star \star} \mathrm{p}<0.01 ;{ }^{* \star *} \mathrm{p}<$ 0.001 .

A

$5-8 \mathrm{~F}$

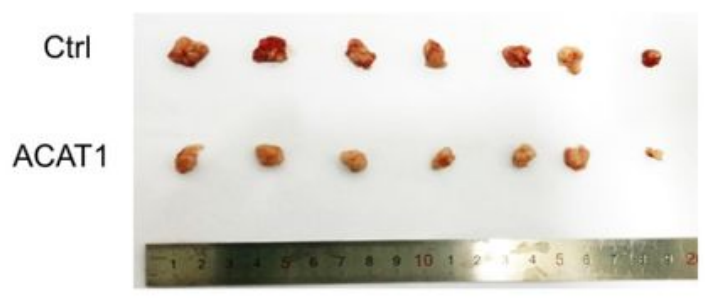

B

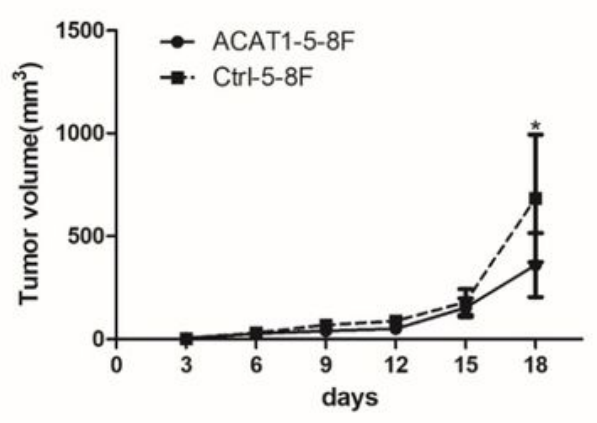

C
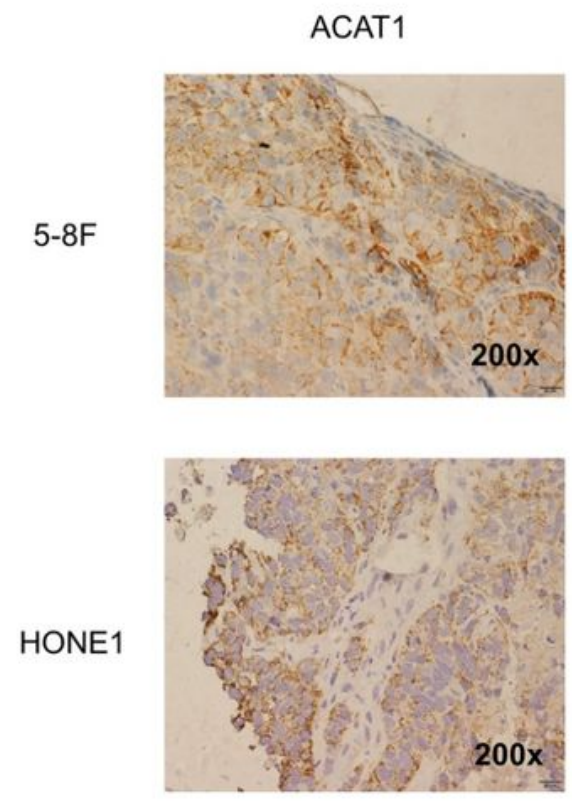

HONE1
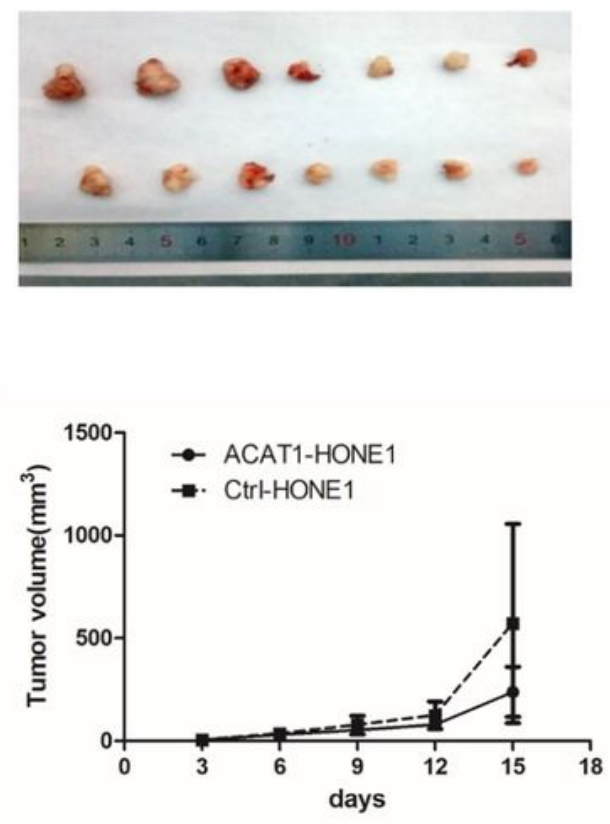

Ctrl
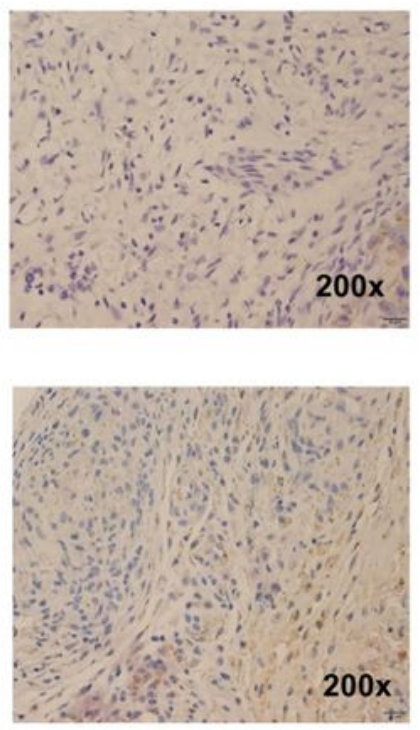

\section{Figure 5}

ACAT1 suppresses tumorigenesis of NPC cells in vivo. A: Xenografts in nude mice from inoculated ACAT1-5-8F/HONE1 and pCMV6-Entry-5-8F cells were removed at day 18 (5-8F) and day 15 (HONE1) B: 
Volume of the tumors measured at $0,3,6,9,12,15,18$ days after inoculation. C: Immunohistochemistry staining was used to determine the expression of ACAT1 in xenografts. ${ }^{*} p<0.05$.

A

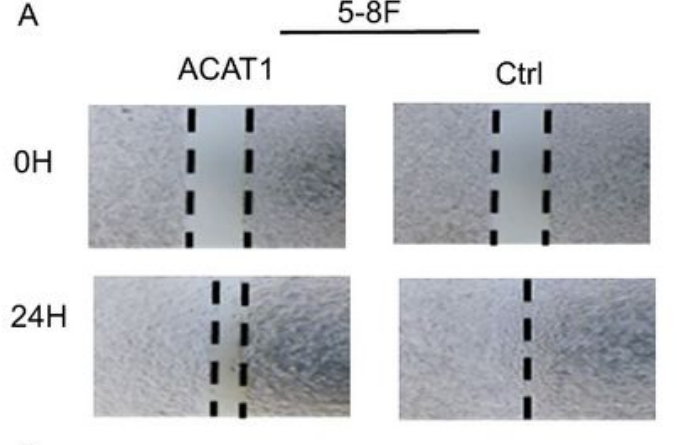

B

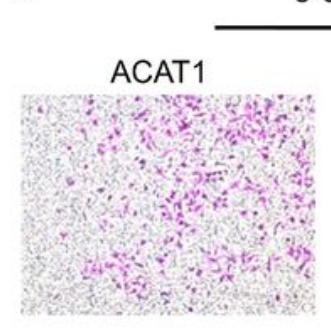

$5-8 \mathrm{~F}$

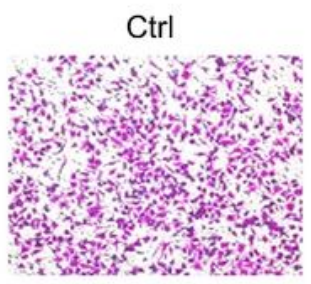

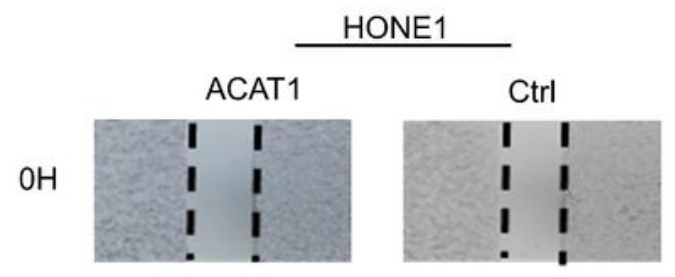

$24 \mathrm{H}$
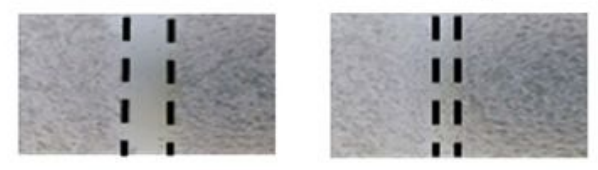

HONE1

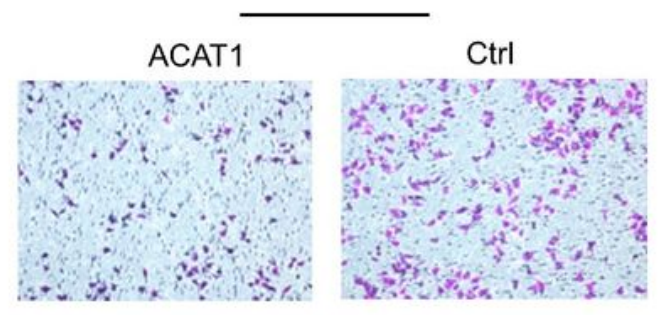

C
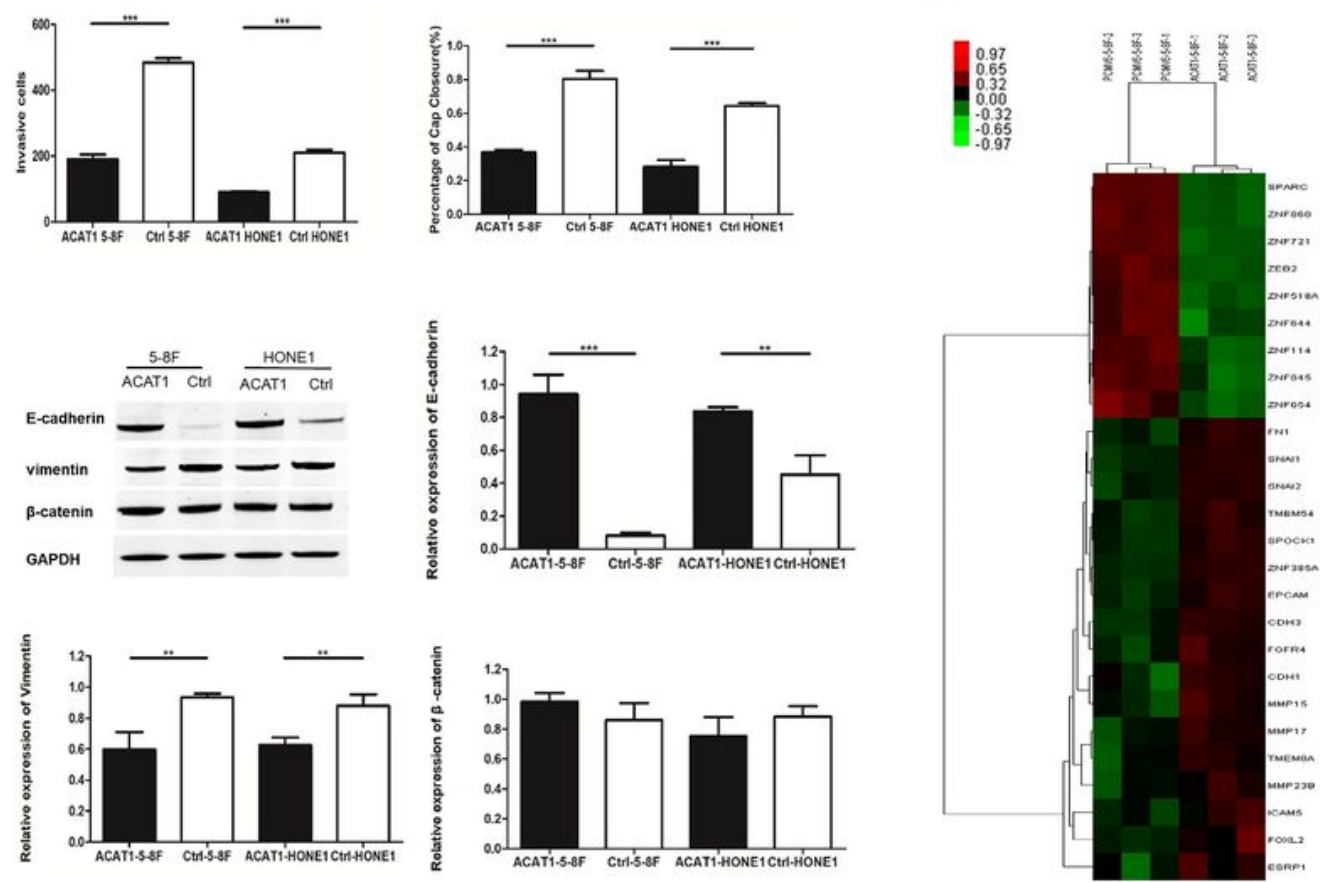

\section{Figure 6}

Overexpression of ACAT1 suppresses the migration and invasion of NPC cell lines in vitro by reversing EMT. (A) Migration of NPC cells stably transfected with ACAT1 or empty vector was examined by a wound healing assay. The gap closure was photographed and measured at 0 and $24 \mathrm{~h}$. The percentage 
of wound width for each sample was calculated by ImageJ software. Magnification $\times 100$. (B) Invasion of NPC cells stably transfected with ACAT1 or empty vector was examined by transwell assay. The blue dots represent the invading cells stained with crystal violet. The number of invading cells was counted and is shown in the bar graph. (C) CDNA microarray data: heatmap showing expression of 26 genes involved in EMT in ACAT1-overexpressing 5-8F cells as compared with pCMV6-Entry-5-8F cells. (D) The expression of E-cadherin, Vimentin and $\beta$-catenin was detected by western blot and quantitated by densitometric scanning. GAPDH was used as an internal control. Data are expressed as means \pm s.d. in a bar graph. $\star \star \star p<0.001$. 
A

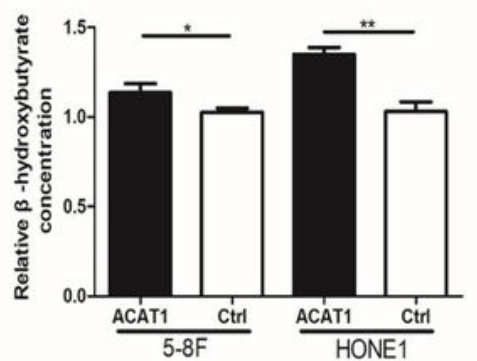

B
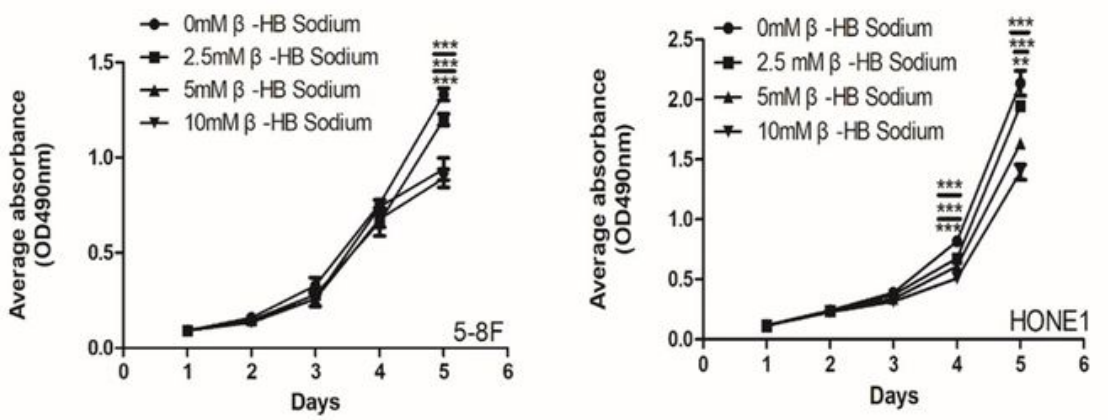

Figure 7

ACAT1 elevates the intracellular level of $\beta$-hydroxybutyrate ( $\beta$-HB) in NPC cells. $(A)$ The relative concentration of intracellular $\beta-\mathrm{HB}$ in ACAT1-5-8F/HONE1 and Ctrl-5-8F/HONE1 cells. (B) MTT assay was performed to measure the proliferation of $5-8 \mathrm{~F}$ and HONE1 cells after $\beta$-HB treatment at $0 \mathrm{mM}, 2.5 \mathrm{mM}, 5$

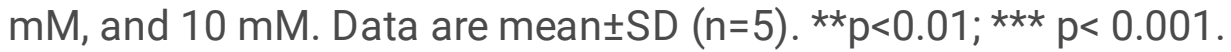




\section{Supplementary Files}

This is a list of supplementary files associated with this preprint. Click to download.

- Supplementaryinformation.docx 